\title{
THE GEOMETRY OF THE TODA EQUATION
}

\author{
DAVID M. J. CALDERBANK
}

\begin{abstract}
I show that solutions of the SU( $\infty)$ Toda field equation generating a fixed Einstein-Weyl space are governed by a linear equation on the Einstein-Weyl space. From this, obstructions to the existence of Toda solutions generating a given Einstein-Weyl space are found. I also give a classification of Einstein-Weyl spaces arising from the Toda equation in more than one way. This classification coincides with a class of spaces found by Ward and hence clarifies some of their properties. I end by discussing the simplest examples.
\end{abstract}

\section{INTRODUCTION}

In [16], Ward showed that solutions $u(x, y, z)$ of the $\mathrm{SU}(\infty)$ Toda field equation $u_{x x}+u_{y y}+\left(e^{u}\right)_{z z}=0$ may be used to define three dimensional Einstein-Weyl spaces. A Weyl space is a conformal manifold $M$ together with a compatible torsion-free connection (called a Weyl connection) and it is said to be Einstein-Weyl iff the symmetric tracefree part of the Ricci tensor of this connection vanishes [7]. Weyl connections on a conformal manifold correspond bijectively to covariant derivatives (called Weyl derivatives) on the density line bundle $L^{1}$, which is the oriented real line bundle whose $n$th power is $\left|\Lambda^{n} T M\right|$ where $n=\operatorname{dim} M$.

A Weyl space may be described by a choice of compatible Riemannian metric $g$ and the connection 1-form $\omega$ of the Weyl derivative on $L^{1}$ relative to the trivialisation of $L^{1}$ determined by the volume form of the metric (so that, for the induced Weyl connection, $D g=-2 \omega \otimes g$ ). In these terms, the Einstein-Weyl space defined by the solution $u$ of the Toda equation may be written:

$$
\begin{aligned}
g & =e^{u}\left(d x^{2}+d y^{2}\right)+d z^{2} \\
\omega & =-u_{z} d z .
\end{aligned}
$$

The Toda equation is a nonlinear integrable system, but very few solutions are known explicitly [1, 3, 田, 13]. Ward found an implicit procedure for generating a family of solutions from axially symmetric harmonic functions $V$. The EinsteinWeyl spaces determined by these implicit solutions are nevertheless completely explicit (in terms of $V$ ) and Ward suggested that "... further investigation is needed to clarify the nature and properties of this family of spaces" 16.

In this paper I show that these Einstein-Weyl spaces are precisely the EinsteinWeyl spaces which can be written in the form (1.1) in at least two inequivalent ways. The key observation is that the solutions of the Toda equation on a fixed

Date: October 2018

1991 Mathematics Subject Classification. 53A30; 53C25.

Key words and phrases. Toda equation, Einstein-Weyl geometry. 
Einstein-Weyl background are essentially given by solutions of a linear system in this Weyl geometry. More precisely, the solutions of this linear system, the "Toda structures", correspond to solutions of the Toda equation on the Einstein-Weyl space up to changes of isothermal coordinates $(x, y)$ and translation of $z$. As a consequence, I show that an Einstein-Weyl space admits at most a four dimensional space of compatible Toda structures, with equality iff the Einstein-Weyl space is Einstein. Furthermore, obstructions to the existence of Toda structures on a given Einstein-Weyl space are found. These obstructions are sufficient to establish which of the local forms of compact Einstein-Weyl spaces (found in [12]) admit Toda structures.

In section 3, I prove that the existence of more than one Toda structure on an Einstein-Weyl space is equivalent to the existence of a conformal vector field of a special type, which I will call an "axial symmetry". This fact is used in section 4 to classify the resulting spaces. The simplest examples are then discussed in the final section.

I work throughout with the density bundles $L^{w}(w \in \mathbb{R})$. A conformal structure may then be defined as an $L^{2}$ valued metric, so that the conformal inner product of vector fields $X, Y$ is $\langle X, Y\rangle \in \mathrm{C}^{\infty}\left(M, L^{2}\right)$. Compatible Riemannian metrics correspond to trivialisations of $L^{1}$, and such a trivialisation is often called a length scale or gauge. When tensoring with a density line bundle, I shall omit the tensor product sign, and sections of $L^{w-1} T M$ or $L^{w+1} T^{*} M$ are called vector fields or 1forms of weight $w$ respectively. The Hodge star operator on an oriented conformal 3-manifold identifies $L^{w}$ with $L^{w+3} \Lambda^{3} T^{*} M$ and $L^{w+1} T^{*} M$ with $L^{w+2} \Lambda^{2} T^{*} M$ and it will be taken to have square -id. For further details see [2, 5]. The results in this paper are local in character, and so, where necessary, vector fields are taken to be nonvanishing and manifolds simply connected.

Acknowledgements. Thanks to Henrik Pedersen and Paul Tod for interesting and helpful discussions.

\section{Toda structures on Einstein-Weyl spaces}

The Einstein-Weyl spaces arising from the Toda Ansatz (1.1) have been characterised by Tod 13 as those which admit a shear-free twist-free geodesic congruence. If $\chi \in \mathrm{C}^{\infty}\left(M, L^{-1} T M\right)$ denotes the weightless unit vector field tangent to this congruence (an oriented foliation with one dimensional leaves) then this means that

$$
D \chi=\tau(i d-\langle\chi, .\rangle \otimes \chi)
$$

where $D$ is the Weyl connection, $\tau$ is a section of $L^{-1}$ and $\langle\chi,$.$\rangle denotes the weight-$ less 1 -form dual to $\chi$ with respect to the conformal structure. In (1.1), the congruence generated by $\partial / \partial z$ has this property and one finds that $2 \tau\langle\chi,\rangle=.-u_{z} d z$. Hence the Weyl derivative $D-2 \tau\langle\chi,$.$\rangle is induced by the Levi-Civita connection$ of $g$ and so the metric $g$ is canonically determined, up to a constant multiple, by the Weyl structure and the congruence [2, 13]. I will denote by $\mu$ the trivialisation of $L^{1}$ corresponding to $g$ and refer to this gauge $\mu$ (unique up to a constant) as the LeBrun-Ward gauge [10, 16]. The congruence $\chi$ determines (in principle) 
the solution of the Toda equation up to the choice of isothermal coordinates $(x, y)$ and affine changes of $z$. Fixing the LeBrun-Ward gauge $\mu$ determines $z$ up to translation. In (1.1), $\tau=-\frac{1}{2} u_{z} \mu^{-1}, \chi=\mu^{-1} \partial / \partial z$ and $\langle\chi,\rangle=.\mu d z$.

The equation (2.1) for $\chi$ is apparently nonlinear, but it actually becomes linear as an equation for the weight $\frac{1}{2}$ vector field $\mathcal{X}=\mu^{1 / 2} \chi$ : explicitly, $D \mathcal{X}=\sigma$ id, for some section $\sigma=\mu^{1 / 2} \tau$ of $L^{-1 / 2}$. Conversely, if $D \mathcal{X}$ is a multiple of the identity, then $\chi=\mathcal{X} /|\mathcal{X}|$ is a shear-free twist-free geodesic congruence and $\mu=|\mathcal{X}|^{2}$ is the LeBrun-Ward gauge.

Although this observation is trivial, it is the key idea behind the results of this paper, and so I should explain its origins. In [10], LeBrun gave a characterisation of the Toda Einstein-Weyl spaces in terms of minitwistor theory [7]. The space of oriented geodesics in a three dimensional Einstein-Weyl space is a complex surface $\mathcal{S}$ containing rational curves ("minitwistor lines") with normal bundle $\mathcal{O}(2)$, and shear-free geodesic congruences correspond to divisors in $\mathcal{S}$ of degree 2 on each minitwistor line. LeBrun noticed that if the congruence is also twist-free, then the corresponding divisor is actually a divisor for $K_{\mathcal{S}}^{-1 / 2}$, where $K_{\mathcal{S}}$ is the canonical bundle of $\mathcal{S}$. After incorporating the choice of homothety factor of the LeBrun-Ward gauge, Toda structures on a fixed Einstein-Weyl space correspond to holomorphic sections of $K_{\mathcal{S}}^{-1 / 2}$. This immediately suggests that a linear equation is involved, and by applying the Penrose transform, following Tsai [15], one finds that sections of $K_{\mathcal{S}}^{-1 / 2}$ correspond to weight $\frac{1}{2}$ vector fields with tracelike derivative. It is then not hard to guess the relationship between such a vector field and $\chi$.

2.1. Definition. A Toda structure on a three dimensional Einstein-Weyl space is a shear-free twist-free geodesic congruence together with a choice of homothety factor for the corresponding LeBrun-Ward gauge.

A Toda structure gives (perhaps only implicitly) a solution of the Toda equation up to changes of isothermal coordinates $(x, y)$ and translation of $z$.

2.2. Proposition. Toda structures correspond to solutions of the following closed linear system for a nonzero weight $\frac{1}{2}$ vector field $\mathcal{X}$ and $a-\frac{1}{2}$ density $\sigma$ :

$$
\begin{aligned}
D \mathcal{X} & =\sigma \text { id } \\
D \sigma & =-\frac{1}{2} F^{D}(\mathcal{X}, .)-\frac{1}{6} \operatorname{scal}^{D}\langle\mathcal{X}, .\rangle
\end{aligned}
$$

where $D$ is the Weyl connection, $F^{D}$ is its curvature on $L^{1}$, and scal ${ }^{D}$ is its scalar curvature, which is a section of $L^{-2}$. Hence Toda structures are parallel sections with respect to a natural connection on $L^{-1 / 2} T M \oplus L^{-1 / 2}$.

Proof. Equation (2.2) has already been established. Differentiating it and skewsymmetrising yields $R_{X, Y}^{D, \frac{1}{2}} \mathcal{X}=\left(D_{X} \sigma\right) Y-\left(D_{Y} \sigma\right) X$, where $R^{D, \frac{1}{2}}$ denotes the curvature of $D$ on $L^{-1 / 2} T M$. Since $D$ is Einstein-Weyl,

$$
R_{X, Y}^{D, \frac{1}{2}}=-\frac{1}{6} \operatorname{scal}^{D}\langle X, .\rangle \triangle Y+\frac{1}{2} F^{D}(X, .) \Delta Y-\frac{1}{2} F^{D}(Y, .) \Delta X+\frac{1}{2} F^{D}(X, Y) \text { id }
$$


where (for any 1-form $\alpha$ and vector fields $X, Y) \alpha \Delta X(Y)=\alpha(Y) X-\langle X, Y\rangle b \alpha$. Equation (2.3) now follows by taking a trace.

2.3. Corollary. An Einstein-Weyl space admits at most a four dimensional space of Toda structures, and hence at most a three parameter family of shear-free twistfree geodesic congruences.

By computing the curvature of the connection

$$
D(\mathcal{X}, \sigma)=\left(D \mathcal{X}-\sigma i d, D \sigma+\frac{1}{2} F^{D}(\mathcal{X}, .)+\frac{1}{6} \operatorname{scal}^{D}\langle\mathcal{X}, .\rangle\right)
$$

on $L^{-1 / 2} T M \oplus L^{-1 / 2}$, one can find obstructions to the existence of Toda structures on Einstein-Weyl spaces. In particular, substituting equation (2.3) back into $R_{X, Y}^{D, \frac{1}{2}} \mathcal{X}=\left(D_{X} \sigma\right) Y-\left(D_{Y} \sigma\right) X$, and using (2.4), yields

$$
F^{D}(X, Y) \mathcal{X}+\langle X, \mathcal{X}\rangle F^{D}(Y)-\langle Y, \mathcal{X}\rangle F^{D}(X)=0
$$

where $F^{D}(X)=b F^{D}(X,$.$) . This condition on \mathcal{X}$ is simply that $\langle\mathcal{X},.\rangle \wedge F^{D}=0$, or equivalently, $\left\langle\mathcal{X}, * F^{D}\right\rangle=0$.

2.4. Proposition. The congruence associated to a Toda structure on an EinsteinWeyl space $(M, D)$ must be orthogonal to $* F^{D}$. Hence $M$ admits a four dimensional space of Toda structures if and only if $F^{D}=0$, i.e., the Einstein-Weyl space is Einstein.

The sufficiency of $F^{D}=0$ follows by verifying that on each of the three Einstein spaces, the Toda structures (given by Tod [13]) do indeed form a four parameter family (where one of the parameters is essentially the homothety factor of the Einstein metric).

The remaining curvature obstructions are obtained by differentiating equation (2.3) and skew-symmetrising. The resulting constraint on $(\mathcal{X}, \sigma)$ is:

$$
\begin{array}{r}
\left(D_{X} F^{D}\right)(Y, \mathcal{X})-\left(D_{Y} F^{D}\right)(X, \mathcal{X})+\frac{1}{3}\left(\langle X, \mathcal{X}\rangle D_{Y} s c a l^{D}-\langle Y, \mathcal{X}\rangle D_{X} s c a l^{D}\right) \\
=F^{D}(X, Y) \sigma .
\end{array}
$$

The Cotton-York curvature of the underlying conformal structure may be defined by $C_{X, Y} Z=\left(D_{X} F^{D}\right)(Y, Z)-\left(D_{Y} F^{D}\right)(X, Z)+\frac{1}{6}\left(\langle X, Z\rangle D_{Y} s c a l^{D}-\langle Y, Z\rangle D_{X} s c a l^{D}\right)$. Hence the above constraint relates $C$ to $D$ scal ${ }^{D}$ and $F^{D}$. Since it is skew in $X, Y$, it is convenient to apply the star operator to obtain

$$
\mathcal{Y}(\mathcal{X}, .)+\frac{1}{6}\left(* D_{\text {scal }}{ }^{D}\right)(\mathcal{X}, .)=\sigma * F^{D}
$$

where $\mathcal{Y}(U, V)=\left\langle *\left(C_{.,} U\right), V\right\rangle$ (which is well known to define a symmetric tracefree tensor). One simple consequence of this is the following refinement of Proposition 2.4.

2.5. Proposition. The congruence associated to a Toda structure on an EinsteinWeyl space $(M, D)$ must be orthogonal to $* F^{D}$ and null with respect to the CottonYork tensor $\mathcal{Y}$. Hence $M$ can only admit a Toda structure if $\mathcal{Y}$ is indefinite on the orthogonal complement of $* F^{D}$. 
To see that this obstruction is nontrivial, I will apply it in the case that the Weyl structure is given by $(g, \omega)$ with $\omega$ dual to a Killing field of $g$. On a compact Einstein-Weyl space, there is a unique compatible metric (up to a constant) with this property, and the Einstein-Weyl structures satisfying this condition have been classified [12]. In order to avoid a case-by-case computation of $\mathcal{Y}, \mathrm{I}$ will derive a general formula.

2.6. Proposition. Suppose $D=D^{g}+\omega$ is Einstein-Weyl with $\omega$ dual to a Killing field of $g$. Then

(i) $D_{X}^{g} F^{D}=\frac{1}{3} \operatorname{scal}^{D} \omega \wedge\langle X,$.$\rangle and$ so $* F^{D}$ is also dual to a Killing field of $g$.

(ii) $\mathcal{Y}(U, V)=\frac{3}{2}\left(\omega(U)\left(* F^{D}\right)(V)+\omega(V)\left(* F^{D}\right)(U)\right)-\left\langle\omega, * F^{D}\right\rangle\langle U, V\rangle$.

Proof. Since $F^{D}=d \omega$ is closed and $D^{g} \omega$ is skew, $D_{X}^{g} F^{D}(Y, Z)=-2\left(R_{Y, Z}^{g} \omega\right)(X)$. The usual formulae for the Ricci tensor of $g$ [5, 12] yield the first result by direct calculation.

Next observe that $D$ scal ${ }^{D}=D^{g} \operatorname{scal}^{D}-2 \operatorname{scal}^{D} \omega$ and that $D_{X} F^{D}(Y, Z)=$ $D_{X}^{g} F^{D}(Y, Z)-F^{D}(\omega \triangle X(Y), Z)-F^{D}(Y, \omega \triangle X(Z))-2 \omega(X) F^{D}(Y, Z)$. Also, by [5], $D^{g}$ Scal ${ }^{D}=3 D^{g}|\omega|^{2}$ and $D^{g} \omega=\frac{1}{2} F^{D}$, which leads to the following formula for $C$ :

$$
\begin{aligned}
C_{X, Y}= & -\omega(X) F^{D}(Y, .)+\omega(Y) F^{D}(X, .) \\
& +\frac{3}{2} F^{D}(b \omega, X)\langle Y, .\rangle-\frac{3}{2} F^{D}(b \omega, Y)\langle X, .\rangle+2 F^{D}(X, Y) \omega .
\end{aligned}
$$

Applying the star operator gives the second formula.

2.7. Corollary. Suppose $D=D^{g}+\omega$ is Einstein-Weyl on $M$ with $\omega$ dual to a Killing field of $g$. Then $(M, D)$ cannot admit a Toda structure unless $* F^{D}$ is orthogonal to $\omega$.

Examining the explicit solutions in [12], one can easily determine for which spaces this holds: in terms of the parameters in Case 1 of [11] (which is the generic case), this condition is $a b c=0$. In particular, among the Berger spheres (given by $b= \pm c$ and $a \neq 0$ ), only the round sphere is Toda, verifying (in another way) the final remarks of [13].

\section{TODA STRUCTURES AND SYMMETRIES}

I turn now to the question: which Einstein-Weyl spaces admit more than a one dimensional family of Toda structures? In the minitwistor space picture, two Toda structures correspond to two holomorphic sections of $K_{\mathcal{S}}^{-1 / 2}$. Their Wronskian, being a section of $K_{\mathcal{S}}^{-1} \otimes T^{*} \mathcal{S} \cong T \mathcal{S}$, is a holomorphic vector field on $\mathcal{S}$. This symmetry of the minitwistor space induces a symmetry of the Einstein-Weyl space.

3.1. Proposition. Suppose $\mathcal{X}_{1}$ and $\mathcal{X}_{2}$ are the weight $\frac{1}{2}$ vector fields of two Toda structures. Then $K=*\left(\mathcal{X}_{1} \wedge \mathcal{X}_{2}\right)$ is a divergence-free twist-free conformal vector field preserving the Weyl connection.

Proof. Differentiating $K$ gives $D K=*\left(\sigma_{1} \mathcal{X}_{2}-\sigma_{2} \mathcal{X}_{1}\right)=\frac{1}{2} d^{D} K$ where $D \mathcal{X}_{i}=$ $\sigma_{i}$ id. This is skew and so $K$ is a divergence-free conformal vector field. Also 
$\langle K,.\rangle \wedge d^{D} K=0$ (since $K$ is orthogonal to $\mathcal{X}_{1}$ and $\mathcal{X}_{2}$ ) so $K$ is twist-free. Finally, to show that $K$ preserves the Weyl connection, it suffices to show that the Lie derivative $\mathcal{L}_{K} D=d \operatorname{tr} D K+F^{D}(K,$.$) of the Weyl derivative on L^{1}$ vanishes. Now $* F^{D}$ is orthogonal to $\mathcal{X}_{1}$ and $\mathcal{X}_{2}$, so $F^{D}(K,)=$.0 , and tr $D K=0$ since $K$ is divergence-free.

Remarkably, the necessary condition of this proposition is also sufficient.

3.2. Theorem. An Einstein-Weyl space has a two dimensional family of Toda structures if and only if it admits a (nonzero) divergence-free, twist-free conformal vector field preserving the Weyl connection.

The necessity is the previous proposition. For the converse, suppose that $K$ is a divergence-free, twist-free conformal vector field preserving the Weyl connection $D$ of an Einstein-Weyl space. Since the result is local, assume $K$ is nonvanishing. Then $D K=\alpha \Delta K$, for some 1-form $\alpha$ with $\alpha(K)=0$. Furthermore, if $D^{|K|}$ is the Weyl derivative corresponding to the trivialisation of $L^{1}$ given by the length of $K$, then $D=D^{|K|}+\alpha$, and so $\mathcal{L}_{K} \alpha=0$. Next note that, since $K$ is twist-free, shear-free and divergence-free, it is surface-orthogonal and the integral surfaces of $K^{\perp}$ are totally geodesic. The above theorem is now an immediate consequence of the following proposition.

3.3. Proposition. Given $D, K, \alpha$ as above, the covariant derivative defined by $D_{X}^{*} \mathcal{X}=D_{X} \mathcal{X}-\alpha(\mathcal{X}) X$ is flat on the bundle of vector fields of weight $\frac{1}{2}$ orthogonal to $K$.

Proof. The curvature of $D^{*}$ is:

$$
\begin{aligned}
R_{X, Y}^{*} \mathcal{X}= & \left(-\frac{1}{6} \operatorname{scal}^{D} X \triangle Y+\frac{1}{2} F^{D}(X, .) \Delta Y-\frac{1}{2} F^{D}(Y, .) \Delta X+\frac{1}{2} F^{D}(X, Y) i d\right)(\mathcal{X}) \\
& -\left(\left(D_{X} \alpha\right)(\mathcal{X})+\alpha(X) \alpha(\mathcal{X})\right) Y+\left(\left(D_{Y} \alpha\right)(\mathcal{X})+\alpha(Y) \alpha(\mathcal{X})\right) X .
\end{aligned}
$$

Now since $K$ is a conformal vector field preserving $D, D_{X}(D K)=R_{X, K}^{D}$. Also $D K=\alpha \Delta K$, so $D_{X}(D K)=\left(D_{X} \alpha+\alpha(X) \alpha\right) \Delta K$. Contracting with $K$ and using the fact that $\alpha(K)=0$ and $\mathcal{L}_{K} \alpha=0$ (i.e., $\left.\left(D_{X} \alpha\right)(K)=-\alpha\left(D_{X} K\right)=\langle K, X\rangle|\alpha|^{2}\right)$ gives

$$
D_{X} \alpha+\alpha(X) \alpha=\frac{1}{2} F^{D}(X)-\frac{1}{6} \operatorname{scal}^{D} X^{\perp}+|\alpha|^{2} X^{\|},
$$

where $X^{\|}$and $X^{\perp}$ denote the components of $X$ parallel and orthogonal to $K$. Substituting this into the formula for $R^{*}$ gives, for $\mathcal{X}$ orthogonal to $K$,

$$
R_{X, Y}^{*} \mathcal{X}=\frac{1}{2} F^{D}(X, Y) \mathcal{X}-\frac{1}{2}\langle\mathcal{X}, Y\rangle b F^{D}(X, .)+\frac{1}{2}\langle\mathcal{X}, X\rangle b F^{D}(Y, .) .
$$

This vanishes for all $X, Y$ because $F^{D}(K,)=$.0 and $\mathcal{X}$ is orthogonal to $K$, so $\langle\mathcal{X},.\rangle \wedge F^{D}=0$.

The parallel sections of $D^{*}$ satisfy $D \mathcal{X}=\alpha(\mathcal{X})$ id and hence give a two dimensional family of Toda structures.

A consequence of this theorem is the following converse to Corollary 2.7. 
3.4. Proposition. Suppose $D=D^{g}+\omega$ is Einstein-Weyl on $M$ with $\omega$ dual to a Killing field of $g$ and that $* F^{D}$ is orthogonal to $\omega$. Then $* F^{D}$ is dual to a divergence-free twist-free conformal vector field preserving the Weyl connection, and so $M$ admits a two dimensional family of Toda structures.

Proof. Let $K=\mu_{g}^{3}\left\langle * F^{D},.\right\rangle$ be the vector field dual to $* F^{D}$ with respect to $g$, where $\mu_{g}$ is the trivialisation of $L^{1}$ determined by $g$. Now by Proposition 2.6, $D^{g} K=-\frac{1}{3} s c a l^{D} \mu_{g}^{3} * \omega$ (here $* \omega$ is viewed as a skew endomorphism). Since $D=$ $D^{g}+\omega, D K=-\frac{1}{3} s c a l^{D} \mu_{g}^{3} * \omega+\omega \Delta K+\omega(K)$ id. Now if $* F^{D}$ is orthogonal to $\omega$ then $\omega(K)=0$ and $-\frac{1}{3} s^{2} a l^{D} \mu_{g}^{3} * \omega=\alpha^{g} \triangle K$ for some 1-form $\alpha^{g}$. Hence $K$ is a divergence-free twist-free conformal vector field, and it preserves the Weyl connection since $F^{D}(K,)=$.0 , by definition.

This result could also be easily established by considering each case in turn (most of which are straightforward). These spaces will feature in the final section.

\section{Einstein-Weyl spaces With an AXIAL Symmetry}

In this section, I will find explicitly all the Einstein-Weyl spaces admitting a two dimensional family of Toda structures. According to the previous section, this is equivalent to classifying the Einstein-Weyl spaces admitting a divergence-free twist-free conformal vector field $K$ preserving the Weyl connection. I will say that these spaces are Einstein-Weyl with an axial symmetry. On such a space, there is a two dimensional family of Toda structures given by the weight $\frac{1}{2}$ vector fields $\mathcal{X}$ orthogonal to $K$ and satisfying $D \mathcal{X}=\alpha(\mathcal{X})$ id, where $D K=\alpha \triangle K$. In particular, $D_{K} \mathcal{X}=\alpha(\mathcal{X}) K=D_{\mathcal{X}} K$, so $\mathcal{L}_{K} \mathcal{X}=0$ and these Toda structures are $K$-invariant.

Pick one such Toda structure $\mathcal{X}$. Then $\alpha(\mathcal{X} /|\mathcal{X}|)$ is the section $\tau$ of $L^{-1}$ given by this Toda structure, and it is only identically zero if $\mathcal{X}$ is a parallel vector field (which can only happen on flat space). As shown by LeBrun [10], $\tau$ is a solution of the abelian monopole equation and applying the Jones and Tod construction 8 to this solution gives a hyperKähler metric with a Killing field $X$ [1], 国. The Toda structure is $K$-invariant, so $K$ lifts to give an additional Killing field of the hyperKähler metric. Since $K$ and $X$ commute, some linear combination must be a triholomorphic Killing field and hence the hyperKähler metric arises via the Gibbons-Hawking Ansatz [6] from a harmonic function on $\mathbb{R}^{3}$. This harmonic function is invariant under a Killing field of $\mathbb{R}^{3}$ and, since $K$ is twist-free, one readily finds that this Killing field must also be twist-free (see [2]). Hence it is a rotational vector field, and the harmonic function is axially symmetric. This proves the following result.

4.1. Theorem. Let $M$ be Einstein-Weyl with an axial symmetry. Then if $M$ is not flat (with translational symmetry), it is one of Ward's Einstein-Weyl spaces 
constructed from an axially symmetric harmonic function on $\mathbb{R}^{3}[16$, and is therefore given explicitly by:

$$
\begin{aligned}
& g=\left(V_{\rho}^{2}+V_{\eta}^{2}\right)\left(d \rho^{2}+d \eta^{2}\right)+d \psi^{2} \\
& \omega=\frac{2 V_{\rho} V_{\eta} d \eta+\left(V_{\rho}^{2}-V_{\eta}^{2}\right) d \rho}{\rho\left(V_{\rho}^{2}+V_{\eta}^{2}\right)}
\end{aligned}
$$

where $\left(\rho V_{\rho}\right)_{\rho}+\rho V_{\eta \eta}=0$.

Note that the monopole on $\mathbb{R}^{3}$ is $V_{\eta}$ : the choice of the integral $V$ of $V_{\eta}$ corresponds to the choice of the quotient of the Gibbons-Hawking metric (see [2]). This freedom involves adding multiples of $\log \rho$ to $V$. Note also that if $V=\log \rho$, then the monopole $V_{\eta}$ degenerates, the Einstein-Weyl space above is $\mathbb{R}^{3}$ itself, and $\partial / \partial \psi$ is the axial symmetry.

The equation for $V$ may be viewed as an equation on $\mathcal{H}^{2}$, by thinking of $V$ as being in the kernel of the conformal Laplacian on $\mathbb{R}^{3} \backslash \mathbb{R}$, which is conformal to $S^{1} \times \mathcal{H}^{2}$. More explicitly, if $v=\rho^{1 / 2} V$, then $v_{\rho \rho}+v_{\eta \eta}=-\frac{1}{4} \rho^{-2} v$ and so $v$ is an eigenfunction of the Laplacian with eigenvalue $\frac{1}{8} s \operatorname{sal}_{\mathcal{H}^{2}}$ on the hyperbolic 2-space $\mathcal{H}^{2}$ with metric $\left(d \rho^{2}+d \eta^{2}\right) / \rho^{2}$.

The original choice of Toda structure $\mathcal{X}$ may be found by rescaling $g$ by $\rho^{2}$ to obtain the Weyl structure in the LeBrun-Ward gauge (again, see [2]):

$$
\begin{aligned}
g_{L W} & =\rho^{2}\left(V_{\rho}^{2}+V_{\eta}^{2}\right)\left(d \rho^{2}+d \eta^{2}\right)+\rho^{2} d \psi^{2} \\
& =\rho^{2}\left(d V^{2}+d \psi^{2}\right)+\left(\rho V_{\eta} d \rho-\rho V_{\rho} d \eta\right)^{2} \\
\omega_{L W} & =-\frac{2 V_{\eta}}{\rho^{2}\left(V_{\rho}^{2}+V_{\eta}^{2}\right)}\left(\rho V_{\eta} d \rho-\rho V_{\rho} d \eta\right) .
\end{aligned}
$$

The 1-form $\rho V_{\eta} d \rho-\rho V_{\rho} d \eta$ is locally exact, and may be integrated explicitly by writing $V=U_{\eta}$ with $U$ axially symmetric and harmonic on $\mathbb{R}^{3}$, so that $z=-\rho U_{\rho}$.

The other Toda structures come from the radial congruences on $\mathbb{R}^{3}$ centred about points on the axis of symmetry. A more democratic approach involves the relationship between these examples and Joyce's construction of torus symmetric scalar flat Kähler metrics [9] from a linear equation on hyperbolic 2-space. Indeed this linear equation, given in Proposition 3.2.1 of [9], is the Cauchy-Riemann form of the equation for $V$, tensored trivially with $\mathbb{R}^{2}$. Ignoring the $\mathbb{R}^{2}$ tensor factor, simply take $x_{1}=\rho, x_{2}=\eta, \phi_{1}=\rho V_{\eta}, \phi_{2}=-\rho V_{\rho}$ to see that Joyce's equation is solved by axially symmetric harmonic functions. However, the advantage of his approach is that the pair $\left(\rho V_{\eta},-\rho V_{\rho}\right)$ is identified with a section $\Phi$ of a square root of the canonical bundle of $\mathcal{H}^{2}$ satisfying an invariant equation. Now the EinsteinWeyl structure may be written

$$
\begin{aligned}
g & =|\Phi|^{2} g_{\mathcal{H}^{2}}+d \psi^{2} \\
\omega & =\Phi^{2} /|\Phi|^{2}
\end{aligned}
$$

and so it does not actually depend upon the choice of coordinates $(\rho, \eta)$ identifying $\mathcal{H}^{2}$ with the upper half plane. Such an identification is given by a choice of a 
point at infinity on the hyperbolic disc and each point in this circle gives a Toda congruence.

Two solutions of Joyce's equation generate a scalar-flat Kähler metric with two Killing fields, and Ward's Einstein-Weyl spaces arise as the quotients by each of these Killing fields. Joyce finds the solution $V=\log \rho$ (which generates $\mathbb{R}^{3}$ ) and superposes it with its image under isometries of hyperbolic 2-space (where these isometries are applied to $\Phi)$. In this way he obtains torus symmetric selfdual conformal structures on $k \mathbb{C} P^{2}$, generalising (for $k \geqslant 4$ ) the torus symmetric examples obtained from the hyperbolic Ansatz of LeBrun 10.

\section{EXAMPLES}

The simplest axially symmetric harmonic functions on $\mathbb{R}^{3}$ are the constant functions and the fundamental solutions. The most trivial solution $V_{\eta}=0, V=\log \rho$ yields $\mathbb{R}^{3}$. If $V_{\eta}=b$ or $V_{\eta}=c / \sqrt{\rho^{2}+\eta^{2}}$ then the Gibbons-Hawking metric is $\mathbb{R}^{4}$ and the triholomorphic Killing field is an infinitesimal translation or selfdual rotation respectively. Hence the Einstein-Weyl spaces obtained are the quotients of $\mathbb{R}^{4}$ by Killing fields (infinitesimal transrotations or rotations) given in 11.

To obtain more complicated examples, one can take linear combinations of fundamental solutions and constant solutions. In this way, one can find the Einstein-Weyl quotients of the Taub-NUT and Eguchi-Hanson metrics, more or less by direct substitution, although more manageable expressions are obtained after transforming the $(\rho, \eta)$ coordinates.

The Taub-NUT solutions are given by $V=a \log \rho+b \eta+c \log \frac{\eta+\sqrt{\rho^{2}+\eta^{2}}}{\rho}$ and it is convenient to set $\rho=r \cos \theta, \eta=r \sin \theta$ so that $\rho V_{\eta}=(b r+c) \cos \theta$ and $\rho V_{\rho}=a-c \sin \theta$. Then

$$
\begin{aligned}
g_{L W} & =\left((b r+c)^{2} \cos ^{2} \theta+(a-c \sin \theta)^{2}\right)\left(d r^{2}+r^{2} d \theta^{2}\right)+r^{2} \cos ^{2} \theta d \psi^{2} \\
\omega_{L W} & =-\frac{2(b r+c)}{r\left((b r+c)^{2} \cos ^{2} \theta+(a-c \sin \theta)^{2}\right)} d\left(-a r \sin \theta+\frac{1}{2} b r^{2} \cos ^{2} \theta+c r\right) .
\end{aligned}
$$

Note that $b c=0$ gives the quotients of $\mathbb{R}^{4}$ mentioned briefly above.

The Eguchi-Hanson solutions are obtained from

$$
V=a \log \rho+\frac{1}{2}(b+c / \varepsilon) \log \frac{\eta-\varepsilon+\sqrt{\rho^{2}+(\eta-\varepsilon)^{2}}}{\rho}+\frac{1}{2}(b-c / \varepsilon) \log \frac{\eta+\varepsilon+\sqrt{\rho^{2}+(\eta+\varepsilon)^{2}}}{\rho},
$$

where $\varepsilon^{2}= \pm 1$ (without loss of generality). When $\varepsilon^{2}=-1$ this is the potential for an axially symmetric circle of charge, while $\varepsilon^{2}=+1$ corresponds to two point sources on the axis of symmetry. These cases are sometimes referred to as EguchiHanson I and II respectively. The former is always incomplete, but its EinsteinWeyl quotients are perhaps more interesting than those of Eguchi-Hanson II. 
Coordinates adapted to these geometries are obtained via $\rho=\sqrt{R^{2}-\varepsilon^{2}} \sin \theta$ and $\eta=R \cos \theta$ so that

and

$$
\begin{aligned}
& \rho V_{\eta}=\frac{(b R+c \cos \theta) \sqrt{R^{2}-\varepsilon^{2}} \sin \theta}{R^{2}-\varepsilon^{2} \cos ^{2} \theta} \\
& \rho V_{\rho}=\frac{a\left(R^{2}-\varepsilon^{2} \cos ^{2} \theta\right)-b\left(R^{2}-\varepsilon^{2}\right) \cos \theta+c R \sin ^{2} \theta}{R^{2}-\varepsilon^{2} \cos ^{2} \theta} .
\end{aligned}
$$

The Toda structure is now given by:

$$
\begin{aligned}
g_{L W}= & \left((a \cos \theta-b)^{2}\left(R^{2}-\varepsilon^{2}\right)+(a R+c)^{2} \sin ^{2} \theta\right)\left(\frac{d R^{2}}{R^{2}-\varepsilon^{2}}+d \theta^{2}\right) \\
& +\left(R^{2}-\varepsilon^{2}\right) \sin ^{2} \theta d \psi^{2} \\
\omega_{L W}=- & \frac{2(b R+c \cos \theta)}{(a \cos \theta-b)^{2}\left(R^{2}-\varepsilon^{2}\right)+(a R+c)^{2} \sin ^{2} \theta} d(-a R \cos \theta+b R-c \cos \theta) .
\end{aligned}
$$

The family given by $a=0, \varepsilon^{2}=-1$ also arises as a quotient of the scalar flat Kähler metric on $S^{2} \times \mathcal{H}^{2}$. If we write this as:

$$
g=\frac{d R^{2}}{R^{2}+1}+\left(R^{2}+1\right) d s^{2}+d \theta^{2}+\sin ^{2} \theta d \phi^{2}
$$

then $K=b \partial / \partial s+c \partial / \partial \phi$ is a Killing field. Coordinates adapted to $K$ are given by $\chi=b s+c \phi, \psi=b \phi-c s$ so that $K$ is a multiple of $\partial / \partial \chi$ and the quotient metric $g-g(K,.) / g(K, K)$ is

$$
\frac{d R^{2}}{R^{2}+1}+d \theta^{2}+\frac{\left(R^{2}+1\right) \sin ^{2} \theta}{b^{2}\left(R^{2}+1\right)+c^{2} \sin ^{2} \theta} d \psi^{2}
$$

This is the same conformal structure as above, and one readily checks that the Weyl structures also agree. Now $S^{2} \times \mathcal{H}^{2}$ is conformal to $\mathbb{R}^{4} \backslash \mathbb{R}$ and so these Weyl structures are globally defined on $S^{3}$ for $b \neq 0$ (since $\partial / \partial s$ is a dilation). Hence, as remarked in [2], these quotients of $\mathbb{R}^{4}$ by dilation plus planar rotation are Toda (although the congruences are not globally defined on $S^{3}$ ). Additionally, the calculations of this section verify explicitly that they are Einstein-Weyl with an axial symmetry, in accordance with Proposition 3.4, and arise from the EguchiHanson I metrics.

\section{REFERENCES}

[1] C. P. Boyer and J. D. Finley, Killing vectors in self-dual Euclidean Einstein spaces, J. Math. Phys 23 (1982) pp. 1126-1130.

[2] D. M. J. Calderbank and H. Pedersen, Selfdual spaces, complex structures, Einstein-Weyl geometry and geodesics, Edinburgh Preprint MS-98-012 (1998).

[3] D. M. J. Calderbank and K. P. Tod, Einstein metrics, hypercomplex structures and the Toda field equation, Edinburgh Preprint MS-98-011 (1998).

[4] J. D. Gegenberg and A. Das, Stationary Riemannian space-times with self-dual curvature, Gen. Relativity Gravitation 16 (1984) pp. 817-829.

[5] P. Gauduchon, Structures de Weyl-Einstein, espaces de twisteurs et variétés de type $S^{1} \times S^{3}$, J. reine angew. Math. 469 (1995) pp. 1-50.

[6] G. W. Gibbons and S. W. Hawking, Gravitational multi-instantons, Phys. Lett. 78B (1978) pp. 430-432. 
[7] N. J. Hitchin, Complex manifolds and Einstein equations, Twistor Geometry and Non-linear Systems (eds H. D. Doebner and T. D. Palev), Lecture Notes in Math. 970 (Springer, Berlin, 1982) pp. 79-99.

[8] P. E. Jones and K. P. Tod, Minitwistor spaces and Einstein-Weyl spaces, Class. Quantum Grav. 2 (1985) pp. 565-577.

[9] D. D. Joyce, Explicit construction of self-dual 4-manifolds, Duke Math. J. 77 (1995) pp. 519 552 .

[10] C. R. LeBrun, Explicit self-dual metrics on $\mathbb{C} P^{2} \# \cdots \# \mathbb{C} P^{2}$, J. Diff. Geom. 34 (1991) pp. 223253.

[11] H. Pedersen and K. P. Tod, Three-dimensional Einstein-Weyl geometry, Adv. Math. 97 (1993) pp. 74-109.

[12] K. P. Tod, Compact 3-dimensional Einstein-Weyl structures, J. London Math. Soc. 45 (1992) pp. 341-351.

[13] K. P. Tod, Scalar-flat Kähler and hyper-Kähler metrics from Painlevé-III, Class. Quantum Grav. 12 (1995) pp. 1535-1547.

[14] K. P. Tod, The SU( $\infty)$-Toda field equation and special four-dimensional metrics, Geometry and Physics (eds J. E. Andersen, J. Dupont, H. Pedersen and A. Swann, Marcel Dekker, New York 1997), pp. 307-312.

[15] C.-C. Tsai, The Penrose Transform for Einstein-Weyl and Related Spaces, PhD Thesis, University of Edinburgh (1996).

[16] R. S. Ward, Einstein-Weyl spaces and SU( $\infty)$ Toda fields, Class. Quantum Grav. 7 (1990) pp. L95-L98.

Department of Mathematics and Statistics, University of Edinburgh, King's Buildings, Mayfield RoAd, Edinburgh EH9 3JZ, Scotland.

E-mail address: davidmjc@maths.ed.ac.uk 